\title{
OS MICROFUNDAMENTOS DO CONSUMO: DE KEYNES ATÉ A VERSÃO MODERNA DA TEORIA DA RENDA PERMANENTE ${ }^{1}$
}

\author{
Flavia Dias Rangel Oreiro ${ }^{3}$
}

\section{INTRODUÇÃO}

Vários economistas já se dedicaram ao estudo da teoria do comportamento do consumidor e à interpretação dos dados sobre consumo e renda. Dentre eles, podemos destacar, em ordem cronológica, o trabalho de John Maynard Keynes, Irving Fisher, Milton Friedman, Franco Modigliani e Robert Hall.

Segundo Keynes, o consumo depende principalmente da renda corrente. Entretanto, seus sucessores argumentam que os consumidores estejam diante de uma decisão intertemporal. Isto é, que a decisão de consumir refere-se à decisão de gastar dinheiro agora ou retê-lo para financiar o consumo futuro. Assim sendo, eles argumentam que a base apropriada para esse estudo de consumo seja a teoria de alocação intertemporal, a qual formaliza os tradeoffs entre consumo presente e consumo futuro.

Este artigo pretende analisar a evolução recente das teorias de consumo, bem como inserir o comportamento do consumidor na ótica intertemporal. O presente artigo está estruturado da seguinte forma: na pri-

${ }^{1}$ Artigo apresentado no IV Encontro da Sociedade Brasileira de Economia Política, realizado na Universidade Federal do Rio Grande do Sul, no período de 1 a 4 de junho de 1999.

${ }^{2}$ Mestre em Economia pela Universidade Federal Fluminense (UFF) e Economista da Petrobras. e-mail: flaviarangel@petrobras.com.br

${ }^{3}$ A autora agradece os comentários e sugestões dos professores Marcelo Cortes Neri (EPGE-FGV/RJ), Antonio Luiz Licha (IE/UFRJ), Jose Luis Oreiro (UFPR) e Gilberto Tadeu Lima (FEA/USP) a uma versão anterior do presente artigo. Eventuais falhas são, no entanto, de minha inteira responsabilidade. 
meira seção, apresentamos a teoria de consumo de Keynes (1936) e discutimos sua relação com os fatos estilizados de Kuznets; na segunda seção, analisamos a contribuição de Irving Fisher à teoria de consumo ao propor o modelo básico de comportamento do consumidor, que baseou as explicações de Modigliani e Friedman para o enigma do consumo; na terceira e na quarta seção, são apresentadas respectivamente a teoria do ciclo da vida de Modigliani (1963) e a teoria da renda permanente de Friedman (1957); a quinta seção analisa a versão moderna da teoria da renda permanente (PIH), também chamada de modelo Hall-Flavin (1978-1981).

\section{A FUNÇÃO CONSUMO KEYNESIANA E OS FATOS ESTILIZADOS DE KUZNETS}

Keynes (1936) dedica os capítulos 8 e 9 da Teoria Geral à análise dos determinantes do consumo. Segundo ele, a quantia que uma família despende em consumo depende:

i) do volume de sua renda;

ii) de fatores objetivos; e

iii) de suas necessidades subjetivas, de propensões psicológicas, dos hábitos dos indivíduos e também dos princípios sobre os quais a renda é dividida entreeles.

Segundo Keynes, os principais fatores objetivos que podem influenciar a propensão a consumir são os seguintes:

i) Uma variação na unidade salarial, uma vez que de acordo com Keynes, o consumo é uma função basicamente da renda real, e não da renda monetária;

ii) Uma variação na diferença entre renda e renda líquida (disponível). Keynes afirma que é na renda disponível que os indivíduos se baseiam ao decidir sua escala de consumo;

iii) Variações nos valores-capital não permitidos no cálculo da renda (líquida) disponível;

iv) Variações na taxa de desconto intertemporal, ou seja, na taxa de troca entre bens presentes e futuros; ${ }^{4}$

\footnotetext{
${ }^{4}$ Vale ressaltar que isso não é o mesmo que a taxa de juros.
} 
v) Variações na política fiscal;

vi) Variações nas expectativas a respeito da relação entre nível de renda presente e futuro.

$\mathrm{Na}$ Teoria Geral, Keynes apresenta vários argumentos valiosos a respeito da decisão de consumo, mas resume a determinação do consumo agregado à chamada lei psicológica fundamental:

The fundamental psychological law, upon which we are entitled to depend with great confidence both a priori from our knowledge of human nature and from the detailed facts of experience, is that men are disposed, as a rule and on the average, to increase their consumption as their income increases, but not by as much as the increase in their income. (KEYNES, 1936, p. 96).

Segundo a lei psicológica fundamental, as variações no nível de renda corrente produziriam variações no nível de consumo corrente, porém em menor proporção. Isso se deve ao fato de que o indivíduo poupa a diferença entre a sua renda efetiva e os gastos necessários para manter o seu padrão habitual de vida. Nas palavras de Keynes:

For a man's habitual standard of life usually has the first claim on his income, and he is apt to save the difference which discovers of his actual income and the expense of habitual standard; or, if he does adjust his expenditure to changes in his income, he will over short periods do so imperfectly. Thus a rising income will often be accompanied by increased saving, and a falling income by decreased saving, on a greater scale at first than subsequently. (KEYNES, 1936, p. 97)

Portanto, a função consumo poderia ser apresentada pela seguinte equação:

$$
C=\bar{C}+c Y ; \quad \text { onde: } \quad \bar{C}>0 \quad e \quad 0<c<1
$$

onde: $\bar{C}$ representa o consumo autônomo; c representa a propensão marginal a consumir. 
Figura $1_{c}$

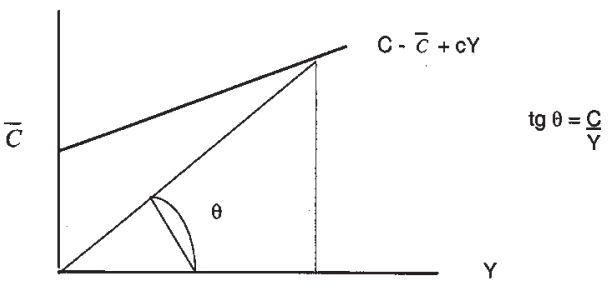

Com base no gráfico acima, quanto mais alto for o nível de renda, maior tenderá a ser a diferença entre a renda e o consumo, isto é, a poupança. Sendo assim, a poupança tenderá a ser maior nas camadas mais ricas da população do que nas camadas mais pobres. Nas palavras de Keynes:

For the satisfaction of the immediate primary needs of a man and his family is usually a stronger motive than the motives towards accumulation, which only acquire effective sway when a margin of comfort has been attained. These reasons will lead, as a rule, to a greater proportion of income being saved as real income increases. (KEYNES, 1936, p. 97).

Portanto, como os indivíduos das camadas mais pobres da população apresentam o seu padrão mínimo de consumo bem próximo do nível de subsistência, eles deixariam as inferências sobre a postergação do poder aquisitivo para o futuro para segundo plano.

Deve-se ressaltar que Keynes (1936) reconhece que as poupanças acumuladas pelas famílias no passado poderiam ser usadas como uma forma de defesa de seu padrão de vida, ou seja, que os indivíduos poderiam poupar por fins puramente precaucionais. Nas palavras de Keynes:

On the other hand, a decline in income due to a decline in the level of employment, if it goes far, may even cause consumption to exceed income not only by some individuals and institutions using up the financial reserves which the accumulated in better times, but also by government, which will be liable, willingly or unwillingly, to run into a budgetary deficit or will provide unemployment relief, for example, out of borrowed money. (KEYNES, 1936, p. 98) 
De acordo com Keynes (1936), existiriam oito motivos de caráter subjetivo em virtude dos quais os indivíduos se abstêm de gastar sua renda, ou seja, prefeririam poupar: ${ }^{5}$

i) A fim de constituir uma reserva para fazer face a contingências imprevistas, isto é, o motivo precaucional;

ii) A fim de preparar-se para uma relação futura prevista entre a renda e as necessidades do indivíduo e sua família, diferente da que existe no momento, como por exemplo no que diz respeito à velhice, à educação dos filhos ou ao sustento das pessoas dependentes, isto é, o motivo ciclo da vida;

iii) A fim de beneficiar-se do juro e da valorização, uma vez que um consumo real maior no futuro é preferível a um consumo imediato mais reduzido, isto é, o motivo substituição intertemporal;

iv) A fim de desfrutar de um gasto progressivamente crescente, satisfazendo a um instinto normal que leva os homens a encarar a perspectiva de um nível de vida que melhore gradualmente, de preferência ao contrário, mesmo que a capacidade de satisfação tenda a diminuir, isto é, o motivo melhoria;

v) A fim de desfrutar de uma sensação de independência ou do poder de fazer algo, mesmo sem idéia clara ou intenção definida da ação específica, isto é, o motivo independência;

vi) A fim de garantir uma massa de manobra para realizar projetos especulativos ou econômicos, isto é, o motivo iniciativa;

vii) A fim de legar uma fortuna, isto é, o motivo herança;

viii) A fim de satisfazer a avareza pura, ou seja, inibir-se de modo irracional, mas persistente, de realizar qualquer ato de despesa como tal, isto é, o motivo avareza.

Pode-se notar facilmente a evidente heterogeneidade nos motivos de poupança. É improvável que uma simples explicação seja suficiente para abranger todos os membros de uma dada população num dado momento, ou para um mesmo indivíduo durante certo período. Podemos intuitivamente observar que as famílias mais ricas apresentam motivações para poupar bem diferentes das famílias mais pobres.

Os motivos citados acima pretendem excluir do consumo uma parte da renda, mas também existem outros motivos correspondentes, que

${ }^{5}$ As designações em itálico são títulos considerados por Browing e Lusardi (1996), mas em geral, quase sempre seguem Keynes. 
às vezes podem provocar um excedente de consumo sobre a renda. Vários dos motivos citados acima que favorecem uma poupança positiva, afetam o comportamento dos indivíduos, tendo sua contrapartida como poupança negativa (despoupança) em data posterior. A título de exemplo, podemos citar a poupança feita para prover na velhice as necessidades de consumo. ${ }^{6}$

Além disso, muitos desses motivos são complementares. Por exemplo, muitas famílias que poupam para a aposentadoria (motivo ciclo da vida), estão concomitantemente guardando reservas financeiras para se proteger de choques de renda ou de consumo (motivo poupança precaucional).

Segundo Keynes (1936), esses motivos subjetivos para poupança podem variar de acordo com vários fatores:

\begin{abstract}
Now the strength of all these motives will vary enormously according to the institutions and organization of the economic society which we presume, according to this habits formed by race, education, convention, religion and current morals, according to present hopes and past experience, according to the scale and technique of capital equipment, and according to the prevailing distribution of wealthy and the established standards of life. (KEYNES, 1936, p. 109).
\end{abstract}

Portanto, Keynes já afirmara, em 1936, que as motivações das famílias quanto às suas decisões de consumo/poupança, (e entre elas o motivo precaucional), poderiam mudar, de acordo com vários fatores como a distribuição de riqueza e os níveis de vida.

Em 1946, Simon Kuznets publicou um estudo sobre o comportamento do consumo e da poupança nos Estados Unidos a partir de séries para o período 1869-1938, obtendo os seguintes "fatos estilizados":

i) Para longos períodos, a relação $\mathrm{C} / \mathrm{Y}$ é estável, ou seja, não apresenta nenhuma tendência a aumentar ou diminuir;

ii) Ao longo de flutuações de curto prazo do nível de renda e de emprego, a relação C/Y cai, quando a economia se expande, ou aumenta quando a economia se contrai, isto é, a propensão a consumir é contracíclica.

${ }^{6}$ Vale ressaltar que o auxílio desemprego financiado por empréstimos deve ser considerado preferivelmente como uma poupança negativa. 
Entretanto, a função consumo keynesiana é incapaz de reproduzir tais fatos estilizados de Kuznets simultaneamente:

Se $C=c Y[\bar{C}=0]$ : a função consumo keynesiana só reproduz o primeiro fato.

Se $C=\bar{C}+c Y ; \quad[\bar{C} \neq 0]$ : a função consumo keynesiana só reproduz o segundo fato.

Figura 2

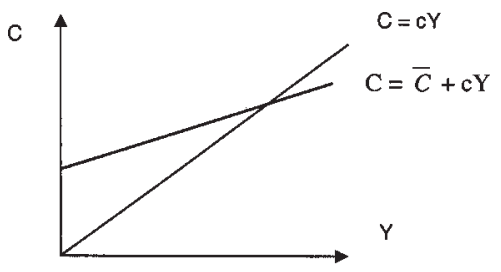

Nos anos 50, Franco Modigliani e Milton Friedman apresentaram explicações para essas constatações aparentemente contraditórias. Contudo, antes de analisarmos como tais autores tentaram resolver esse enigma do consumo, veremos a contribuição de Irving Fisher à teoria de consumo, já que tanto a teoria da renda permanente de Friedman, quanto a teoria de ciclo da vida de Modigliani, se basearam na teoria proposta anteriormente por Irving Fisher.

\section{O MODELO BÁSICO DO COMPORTAMENTO DO CONSUMIDOR: UMA CONTRIBUIÇÃO COM BASE EM IRVING FISHER}

Apresentaremos a seguir as principais hipóteses do modelo básico de comportamento do consumidor:

1) O consumidor possui preferências a respeito da quantidade de bens que irá consumir ao longo de sua vida. Essas preferências podem ser representadas por meio de uma função utilidade do seguinte tipo:

$\mathrm{U}\left(\mathrm{C}_{0}, \mathrm{C}_{1}, \ldots, \mathrm{C}_{\mathrm{t}}, \ldots, \mathrm{C}_{\mathrm{T}}\right)$

2) O consumidor possui informação perfeita ("perfect foresight") a respeito dos rendimentos que irá obter ao longo da sua vida, ou seja, ele conhece os rendimentos: $\mathrm{Y}_{0}, \ldots, \mathrm{Y}_{\mathrm{t}}, \ldots, \mathrm{Y}_{\mathrm{T}}$; 
3) O consumidor não está "restrito por liquidez", ou seja, num determinado período ele pode consumir mais do que ganha, uma vez que o indivíduo pode financiar o seu excesso de consumo sobre a renda com empréstimos, ou pode consumir menos do que ganha, acumulando assim, uma poupança positiva que utiliza na compra de ativos financeiros;

4) Os indivíduos não recebem e nem deixam heranças. Além disso, suporemos que os indivíduos não podem morrer deixando dívidas (condição de No-Ponzi-Game).

Para um indivíduo que vive dois períodos, temos:

$$
\begin{aligned}
& S_{1}=Y_{1}-C_{1} ; \quad \therefore \quad C_{1}=Y_{1}-S_{1} \\
& C_{2}=Y_{2}+S_{1}(1+r)
\end{aligned}
$$

Substituindo (3) em (4), temos:

$$
C_{2}=Y_{2}+\left(Y_{1}-C_{1}\right)(1+r)
$$

Portanto,

$$
C_{1}+\frac{C_{2}}{(1+r)}=Y_{1}+\frac{Y_{2}}{(1+r)}
$$

A equação acima é a restrição orçamentária intertemporal dos indivíduos, que diz que o valor presente do consumo deve ser igual ao valor presente dos rendimentos;

5) As preferências dos indivíduos a respeito de $\left(\mathrm{C}_{1}, \mathrm{C}_{2}, \ldots, \mathrm{C}_{\mathrm{T}}\right)$ apresentam as seguintes características:

a) São estritamente convexas, ou seja, tomando duas cestas de consumo, $\mathrm{C}_{\mathrm{A}}=\left(\mathrm{C}_{1}{ }_{1}^{\mathrm{A}}, \mathrm{C}_{2}{ }^{\mathrm{A}}\right)$ e $\mathrm{C}_{\mathrm{B}}=\left(\mathrm{C}_{2}{ }^{\mathrm{B}}, \mathrm{C}_{2}{ }^{\mathrm{B}}\right)$, tal que :

$$
\mathrm{C}_{\mathrm{A}} \sim \mathrm{C}_{\mathrm{B}} \Rightarrow \lambda \mathrm{C}_{\mathrm{A}}+(1-\lambda) \mathrm{C}_{\mathrm{B}}>\mathrm{C}_{\mathrm{A}}
$$

onde: $\mathrm{C}_{1}$ : consumo no primeiro período

$\mathrm{C}_{2}$ : consumo no segundo período

Essa hipótese nos diz que os meios são preferíveis aos extremos, isto é, os indivíduos preferem consumir um pouco no primeiro e no segundo períodos da vida, do que especializar o seu consumo em um dos dois períodos. 
Figura 3

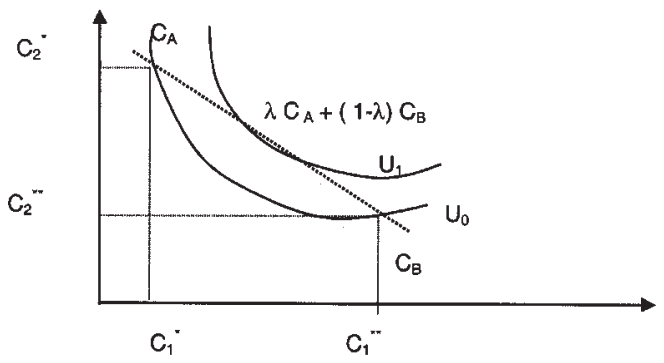

b) São aditivamente separáveis, isto é, a utilidade do consumo em um certo período só depende do consumo daquele período:

$$
U\left(C_{1}, C_{2}\right)=v\left(C_{1}\right)+u\left(C_{2}\right)
$$

c) O indivíduo é impaciente intertemporalmente, ou seja, o indivíduo prefere consumir no presente do que consumir no futuro, o que faz com que ele desconte a utilidade de consumo a uma dada taxa de desconto intertemporal $\theta$, estritamente subjetiva:

$$
U\left(C_{1}, C_{2}\right)=v\left(C_{1}\right)+\frac{1}{(1+\theta)} U\left(C_{2}\right)
$$

d) As preferências podem ser representadas por uma função log-neperiana do tipo:

$$
U\left(C_{1}, C_{2}\right)=\ln C_{1}+\frac{1}{(1+\theta)} \ln C_{2}
$$

Dadas essas hipóteses, o problema do consumidor consiste em escolher o $\operatorname{par}\left(C_{1}^{*}, C_{2}^{*}\right)$, tal que :

$$
\begin{aligned}
& \max \ln C_{1}+\frac{1}{(1+\theta)} \ln C_{2} \\
& \text { s.a. } C_{1}+\frac{C_{2}}{(1+r)}=Y_{1}+\frac{Y_{2}}{(1+r)}
\end{aligned}
$$


Esse problema pode ser resolvido da seguinte forma:

Figura 4

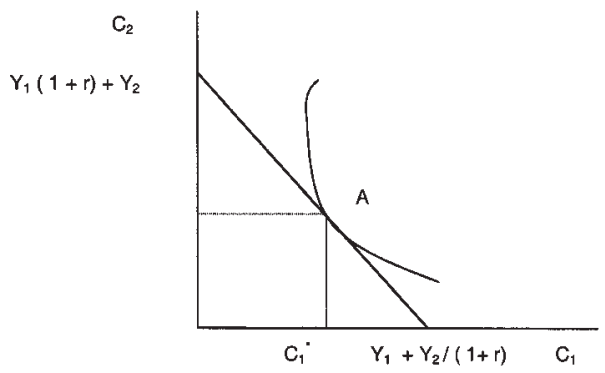

Dessa maneira, temos a seguinte solução algébrica:

$$
\underset{C_{1}, C_{2}}{\max } \mathfrak{I}=\ln C_{1}+\frac{\ln C_{2}}{(1+\theta)}-\lambda\left(C_{1}+\frac{C_{2}}{(1+r)}-Y_{1}-\frac{Y_{2}}{(1+r)},\right.
$$

Condição de Primeira Ordem (C. P. O.):

$$
\begin{aligned}
& \frac{\partial \mathfrak{I}}{\partial C_{1}}=\frac{1}{C_{1}}-\lambda=0 \\
& \frac{\partial \mathfrak{I}}{\partial C_{2}}=\frac{1}{(1+\theta)} \frac{1}{C_{2}}-\frac{\lambda}{(1+r)}=0
\end{aligned}
$$

Colocando $\lambda$ em evidência, temos:

$$
\lambda=\frac{1}{C_{1}} ; \quad e \quad \lambda=\frac{(1+r)}{(1+\theta)} \frac{1}{C_{2}}
$$

Portanto,

$$
C_{1}=C_{2} \frac{(1+\theta)}{(1+r)}
$$

Assim sendo, podemos obter a "condição Keynes-Ramsey":

$$
\frac{C_{2}(1+\theta)}{C_{1}}=(1+r)
$$


Em equilíbrio, a taxa marginal de substituição entre o consumo presente e o consumo futuro, $\frac{C_{1}}{C_{2}(1+\theta)}=\frac{1}{(1+r)}$, deve ser igual a inclinação da restrição orçamentária. Isso vai ocorrer no ponto em que a restrição orçamentária for tangente à curva de indiferença mais elevada possível. (ver gráfico anterior).

Figura 5

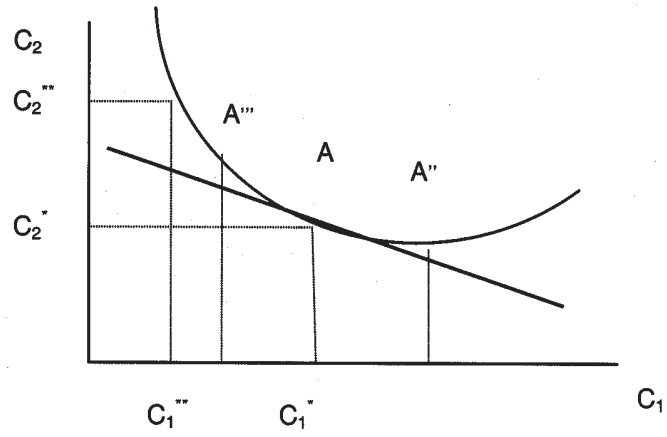

Podemos entender a intuição econômica desse resultado da seguinte forma:

Se $C_{1}^{*}=C_{2}^{*}=1$, então, no ponto $\mathrm{A}:(1+\theta)=(1+r)$

Se $(1+\theta)>(1+r)$, então, o indivíduo para abrir mão de um infinitésimo de consumo presente, deseja obter um aumento de consumo futuro, maior do que aquele que o mercado paga (A'). O indivíduo, dessa forma, valoriza muito $\mathrm{C}_{1}$, pois quando aumenta $\mathrm{C}_{1}$, diminui $\mathrm{C}_{2}$.

Por outro lado, se $(1+\theta)<(1+r)$, então no ponto A", diminuindo $\mathrm{C}_{1}$, aumenta $\mathrm{C}_{2}$.

Temos ainda, de acordo com a condição Keynes-Ramsey:

$$
C_{2}=C_{1} \frac{(1+r)}{(1+\theta)}
$$

Ou seja, a evolução do consumo ao longo do tempo depende fundamentalmente da relação entre $\mathrm{r}$ e $\theta$ :

- Se $r>\theta \Rightarrow$ o consumo cresce ao longo do tempo;

- Se $r<\theta \Rightarrow$ o consumo diminui ao longo do tempo;

- Se $r=\theta \Rightarrow$ o consumo permanece constante ao longo do tempo. 
Figura 6

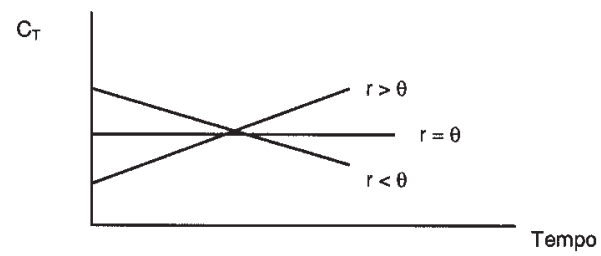

O modelo de escolha intertemporal tem como implicação principal a ausência de relação estrutural entre C e Y corrente. De fato, substituindo (17) na restrição orçamentária:

$$
\begin{aligned}
& C_{1}\left[1+\frac{1}{(1+\theta)}\right]=\quad Y_{1}+\frac{Y_{2}}{(1+r)} \\
& C_{1}=\frac{1}{1+\frac{1}{(1+\theta)}}\left\{Y_{1}+\frac{Y_{2}}{(1+r)}\right\}=K\left\{Y_{1}+\frac{Y_{2}}{(1+r)}\right\}
\end{aligned}
$$

O consumo corrente depende não só da renda corrente, mas também da renda "esperada" para o futuro. Se $\mathrm{Y}_{1}$ aumenta, mas $\mathrm{Y}_{2}$ diminui, de maneira que o valor presente dos rendimentos não se altera e $\mathrm{C}_{1}$ permanece constante. Em outras palavras, as variações puramente temporárias na renda não apresentam nenhum efeito sobre a decisão de consumo, embora influenciem a poupança. Trata-se de um resultado totalmente contrário ao que seria obtido pela função consumo keynesiana. De fato, esta propõe a existência de uma relação estrutural entre $\mathrm{C}$ e $\mathrm{Y}$.

\section{O MODELO CICLO DA VIDA DE MODIGLIANI}

O modelo ciclo da vida baseia-se na idéia de que o consumo de um determinado período não depende da renda corrente, mas da renda auferida ao longo de toda a vida economicamente ativa (life-time earnings). ${ }^{7} \mathrm{De}$ acordo com as hipóteses do ciclo da vida, a renda dos indivíduos tende a sofrer flutuações sistemáticas ao longo da vida. Dessa forma, o comporta-

${ }^{7}$ Como referências a respeito da teoria do ciclo da vida, podemos citar DEATON, A. Understanding Consumption. Oxford University Press, 1992. 
mento da poupança seria determinado pelo estágio do ciclo da vida em que o indivíduo se encontra.

A figura abaixo caracteriza os diversos estágios do ciclo da vida de um consumidor:

1) No estágio I, referente à juventude, a renda dos indivíduos é baixa, e tais indivíduos, geralmente, contraem dívidas porque sabem que ganharão rendas maiores no futuro;

2) No estágio II, referente à meia idade, a renda atinge um pico e os indivíduos pagam as dívidas contraídas no estágio I, além de pouparem para a velhice;

3) No estágio III, referente à velhice, a renda tende a zero, e os indivíduos consomem toda a poupança acumulada.

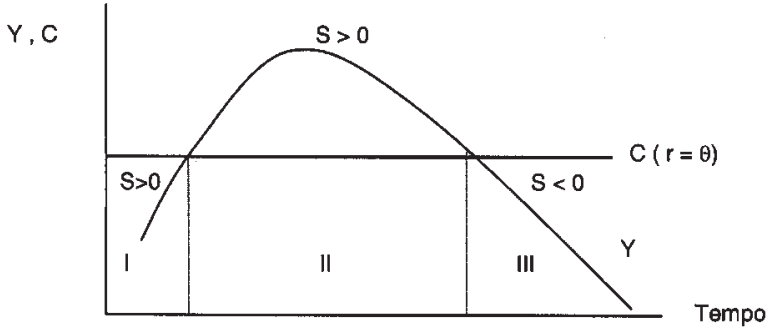

Figura 7

Sendo assim, as flutuações da renda corrente teriam impacto unicamente sobre a poupança dos indivíduos, e não sobre a sua decisão de consumo. Em outras palavras, a decisão de consumo seria unicamente determinada pelo valor presente dos rendimentos ao longo da vida e pela relação entre $r$ e $\theta$.

\section{A TeOria da Renda PERManente de Milton FRIEDMAN ${ }^{8}$}

Friedman (1957) parte da premissa de que as famílias desejam nivelar o consumo ao longo do tempo, isto é, o consumo não deve ser influenciado pelas variações puramente transitórias nos rendimentos das famílias. As famílias pautariam suas decisões de consumo com base na sua renda permanente, ou seja, o nível constante de renda que proporciona à

${ }^{8}$ As idéias referentes à teoria da renda permanente foram expostas por FRIEDMAN, M. A Theory of the Consumption Function. Princeton University Press, 1957. 
família, a mesma restrição orçamentária intertemporal que ela teria no caso de um fluxo de renda flutuante.

A restrição orçamentária para o caso de renda flutuante seria:

$C_{1}+\frac{C_{2}}{(1+r)}=Y_{1}+\frac{Y_{2}}{(1+r)}$

Pela definição de renda permanente, temos:

$$
\begin{aligned}
& Y_{1}+\frac{Y_{2}}{(1+r)}=Y_{P}+\frac{Y_{P}}{(1+r)} \\
& Y_{P}\left[1+\frac{1}{(1+r)}\right]=Y_{1}+\frac{Y_{2}}{(1+r)} \\
& Y_{P}=\left[\frac{1+r}{2+r}\right]\left[Y_{1}+\frac{Y_{2}}{(1+r)}\right]
\end{aligned}
$$

O caso friedmaniano é caracterizado por:

i) $\mathrm{C}_{1}=\mathrm{C}_{2}=\bar{C}$;

ii) $\mathrm{r}=\delta$;

iii) $U\left(C_{1}, C_{2}\right)=\ln C_{1}+\frac{1}{(1+\delta)} \ln C_{2}$

Portanto, o problema do consumidor consiste:

$$
\begin{aligned}
& \max _{C_{1}, C_{2}} \mathfrak{I}=\ln C_{1}+\frac{1}{(1+\delta)} \ln C_{2}-\lambda\left[C_{1}+\frac{C_{2}}{(1+r)}-\frac{2+r}{1+r} Y_{P}\right] \\
& \frac{\partial \mathfrak{I}}{\partial C_{1}}=\frac{1}{C_{1}}-\lambda=0 \\
& \frac{\partial \mathfrak{I}}{\partial C_{2}}=\frac{1}{(1+r)} \frac{1}{C_{2}}-\frac{\lambda}{(1+r)}=0
\end{aligned}
$$

Portanto:

$$
C_{1}=C_{2}=\bar{C}
$$


Dessa forma,

$$
\begin{aligned}
& \bar{C}+\frac{\bar{C}}{(1+r)}=Y_{P}+\frac{Y_{P}}{(1+r)} \\
& \bar{C}\left[1+\frac{1}{(1+r)}\right]=Y_{P}\left[1+\frac{1}{(1+r)}\right] ; \\
& \bar{C}=Y_{P}
\end{aligned}
$$

De acordo com Friedman, a poupança consistiria na diferença entre a renda corrente e a renda permanente. Em outras palavras, os indivíduos tendem a poupar as variações puramente transitórias em suas rendas, não tendo estas influências sobre a sua decisão de consumo.

Sendo assim, quando os indivíduos se confrontam com uma variação na sua renda, procurarão determinar se essa variação é permanente ou transitória. Se a variação for transitória, eles ajustarão sua poupança de forma a manter seu padrão de consumo estável.

Segundo Friedman, a renda corrente seria o somatório de dois elementos: a renda permanente e a renda temporária. Enquanto a renda permanente seria a parte da renda que as pessoas esperam manter no futuro, a renda temporária seria a parte da renda que eles não esperam manter no futuro. Em outras palavras, a renda permanente seria a renda média e a renda transitória seria o desvio em relação a essa média.

Friedman considera que o enigma do consumo ocorrera devido à função consumo keynesiana ter levado em conta a renda corrente ao invés da renda permanente. Além disso, segundo Friedman, a propensão média a consumir depende da razão entre a renda permanente e a renda corrente. ${ }^{9}$ Quando a renda corrente estivesse temporariamente acima da renda permanente, a propensão média a consumir cairia, mas quando a renda corrente estivesse abaixo da renda permanente, a propensão média a consumir aumentaria temporariamente.

Analisando os dados de renda familiar, Friedman ainda notou que as famílias de mais alta renda permanente teriam proporcionalmente um consumo mais elevado. Quando todas as variações na renda corrente decorressem do componente permanente, não haveria diferenças entre as famílias quanto à propensão média a consumir. Entretanto, as variações na renda também podem ser provocadas pela renda transitória, e as famílias com ren-

\footnotetext{
${ }^{9}$ A propensão média a consumir seria igual a $\mathrm{C} / \mathrm{Y}=\mathrm{K} \mathrm{Y}_{\mathrm{P}} / \mathrm{Y}$.
} 
das transitórias mais altas não teriam um maior consumo. Portanto, as famílias com rendas mais elevadas teriam menor propensão a consumir.

Já a análise das séries temporais mostra que as flutuações de ano para ano são dominadas pela renda transitória. Assim sendo, os anos com rendas mais elevadas apresentariam baixa propensão média a consumir. Em períodos longos, a variação da renda seria provocada por mudanças no componente permanente. Nas longas séries temporais, a propensão média a consumir deveria ser constante.

\section{A VERSÃO MODERNA dA TEORIA dA RENDA PERMANENTE: O MODELO HALL-FLAVIN (1978-1981).}

Recentemente, alguns autores estenderam a idéia de otimização do uso da informação utilizada na formação de expectativas à esfera de consumo, baseados na crítica de Lucas à utilização indiscriminada de distribuições fixas de defasagens. Sendo assim, tais autores aplicaram o método de expectativas racionais aos modelos ciclo da vida/ renda permanente. Dessa forma, os consumidores resolveriam o modelo relevante da economia, utilizando toda a informação disponível para prever as suas rendas futuras na hora de decidirem o seu padrão de compras.

O modelo Hall-Flavin é um modelo intertemporal padrão, chamado muitas vezes de modelo de equivalência de certeza, que assume algumas hipóteses específicas:

i) Os agentes apresentam funções utilidade aditivas intertemporalmente;

ii) Os mercados de crédito são perfeitos;

iii) Os agentes maximizam a utilidade esperada;

iv) Os agentes formam expectativas racionais;

v) As funções utilidade são quadráticas;

iv) a taxa de juros igual à taxa de desconto intertemporal.

Na verdade, o problema do consumidor consistiria em:

$$
\begin{aligned}
& \max E_{T} \sum_{i=0}^{\infty}(1+\theta)^{-1}\left(a C_{t+i}-\frac{b}{2} C_{t+i}^{2}\right) \\
& \text { sujeito à } \\
& A_{t+i}=(1+r)\left(A_{t}+Y_{t}-C_{t}\right)
\end{aligned}
$$


Portanto, como a condição de primeira ordem para o caso mais geral nos dá a equação de Euler, temos:

$$
U^{\prime}\left(C_{t}\right)=\frac{E_{T}(1+r)}{(1+\theta)} U^{\prime}\left(C_{t+1}\right)
$$

No caso quadrático, em que $\mathrm{r}=\theta=0$, temos;

$$
\begin{aligned}
& a-b C_{t}=E_{t}\left\{a-b C_{t+1}\right\} \\
& a-b C_{t}=a-b E_{t} C_{t+1} \\
& C_{t}=E_{t} C_{t+1} \\
& \text { Como } C_{t}=E_{t}\left\{C_{t+1}\right\} \text { ou ex-post, então; } \\
& C_{t+1}=C_{t}+v_{t} .
\end{aligned}
$$

Hall (1978) concluiu que o consumo presente seguiria um passeio aleatório com uma tendência. A única variável relevante que influenciaria o consumo presente seria o consumo defasado com um período. Isto é, o melhor previsor do consumo futuro seria o consumo presente ajustado por uma tendência. Assim sendo, as mudanças não-antecipadas nos ativos, na renda, nos preços ou em qualquer outra variável relevante deslocariam o consumo da trajetória anteriormente delineada.

Entretanto, Hall não conseguiu refutar a relevância do poder preditivo de um dos componentes defasados da riqueza total na determinação do consumo agregado, pois as mudanças defasadas de até um trimestre no preço das ações negociadas em bolsa revelaram significativas influências sobre o consumo corrente. Logo, isso equivaleria à rejeição empírica da hipótese simples de passeio aleatório no âmbito do consumo. Contudo, Hall reconcilia a validade de sua hipótese reconhecendo a ocorrência de fricções no ajuste do consumo às mudanças da renda permanente, captadas pelas flutuações nos preços das ações.

Em resumo, assumindo expectativas racionais , a expectativa para o consumo corrente $\left(\mathrm{C}_{\mathrm{t}}\right)$, formada no período $(\mathrm{t}-1)$ estaria exclusivamente condicionada ao valor do consumo defasado de um período $\left(\mathrm{C}_{\mathrm{t}-1}\right)$. Logo, o consumo seguiria o passeio aleatório:

$$
\mathrm{C}_{\mathrm{t}}=\mathrm{C}_{\mathrm{t}-1}+\mathrm{v}_{\mathrm{t}}
$$

onde: $\mathrm{v}_{\mathrm{t}}$ independe das variáveis anteriores, constituindo o componente não antecipado do consumo corrente justificado pela revisão das expectativas da renda permanente engendrada por variáveis a ela associadas, como a renda corrente. 


\title{
CONCLUSÃO
}

Este artigo analisou a evolução recente das teorias de consumo desde a teoria de Keynes (1936) até a versão moderna da teoria da renda permanente de Hall-Flavin (1978), enfatizando os micro-fundamentos da função consumo de Keynes, Modigliani, Friedman e Hall-Flavin.

\section{RESUMO}

Este artigo pretende analisar a evolução recente das teorias de consumo, enfatizando os micro-fundamentos do consumo, desde Keynes (1936) até a versão moderna da teoria da renda permanente de HallFlavin (1978-1981), bem como inserir o comportamento do consumidor numa ótica intertemporal. Nesse contexto, são discutidas: a teoria de consumo de John Maynard Keynes (1936); os fatos estilizados de Simon Kuznets (1940); a contribuição de Irving Fisher à teoria de consumo ao propor o modelo intertemporal básico de comportamento do consumidor; a teoria da renda permanente de Milton Friedman (1957) e a teoria do ciclo da vida de Franco Modigliani (1963), as quais se baseiam no arcabouço teórico de Fisher para explicar o "enigma do consumo"; e finalmente, a chamada "versão moderna da teoria da renda permanente" de Hall - Flavin (1978-81), que aplica o método de expectativas racionais aos modelos ciclo-da vida/ renda permanente.

Palavras-chave: microfundamentos do consumo, renda corrente, renda permanente.

\begin{abstract}
The objective of the present article is to analyze the recent evolution of the theories of the consumption function, with a special emphasis over the micro foundations of the consumption decision, since Keynes (1936) until the modern version of the permanent income hypothesis of Hall and Flavin (1978-1981). In this setting we will discuss the following issues: the consumption function theory of John Maynard Keynes, the stylized facts of Kusnets, the Irving Fisher contribution to the consumption function theory by his proposal of an intertemporal framework to analyze consumer behavior; Milton Friedman's theory of permanent income and Modigliani's life-cycle
\end{abstract}


hypothesis. All these theories are based upon Irving Fisher framework to explain the "consumption enigma". Finally we will analyze the Hall-Flavin version of the permanent income hypothesis, which applies the rational expectations method to the models of aggregate consumption based on the life-cycle/permanent income hypothesis. Key-words: micro-foundations of the consumption function, current income, permanent income.

\section{ANEXO: DEMONSTRAÇÃO PARA O CASO DA FUNÇÃO UTILIDADE QUADRÁTICA:}

Como $\mathrm{r}=\theta$ e a utilidade é quadrática, temos que:

$$
C_{t}=E_{t} C_{t+1}
$$

De acordo com a lei das expectativas iterativas:

$$
\begin{aligned}
& E_{t} C_{t+2}=E_{t}\left(E_{t+1} C_{t+2}\right)=E_{t}\left(C_{t+1}\right)=C_{t} \\
& \text { Logo: } E_{t} C_{t+i}=C_{t}, \forall i
\end{aligned}
$$

Integrando a restrição orçamentária para frente, obtemos:

$$
A_{t}=C_{t}-Y_{t}+\frac{A_{t+1}}{(1+r)}=C_{t}-Y_{t}+\frac{C_{t+1}-Y_{t+1}}{(1+r)}+\frac{A_{t+2}}{(1+r)}
$$

Portanto, generalizando a restrição orçamentária, temos:

$$
A_{t}=\sum_{i=0}^{T} \frac{C_{t+i}}{(1+r)^{i}}-\sum_{i=0}^{T} \frac{Y_{t+i}}{(1+r)^{i}}+\frac{A_{T}}{(1+r)^{T}}
$$

Deve-se ressaltar que, de acordo com a condição de jogo no ponzi, limita-se o volume de despoupança à restrição orçamentária do agente:

$$
\lim _{i \rightarrow \infty} \frac{A_{t+i}}{(1+r)^{i}}=0
$$

Dessa forma, se passarmos as expectativas na restrição orçamentária, e se utilizarmos a expressão : $E_{t} C_{t+i}=C_{t}, \forall i$, temos: 


$$
A_{t}=\sum_{i=0}^{\infty} \frac{C_{t}-E_{t} Y_{t+1}}{(1+r)^{i}}
$$

Contudo, ao calcularmos esse somatório, utilizando a fórmula da soma de uma progressão geométrica, obtemos:

$$
C_{t} \sum_{i=0}^{\infty} \frac{1}{(1+r)^{i}}=C_{t} \frac{1}{1-\frac{1}{(1+r)}}=C_{t} \frac{(1+r)}{r}
$$

Logo, ao substituirmos a expressão acima na restrição orçamentária, temos:

$$
\begin{aligned}
& A_{t}=\sum_{i=0}^{\infty} \frac{C_{t}}{(1+r)}-E_{t} \sum_{i=0}^{\infty} \frac{Y_{t+i}}{(1+r)^{i}} \\
& A_{t}=\frac{C_{t}(1+r)}{r}-E_{t} \sum_{i=0}^{\infty} \frac{1}{(1+r)^{i}} Y_{t+i}
\end{aligned}
$$

Desse modo, rearranjando os termos:

$$
C_{t}=\left(\frac{r}{1+r}\right) A_{T}+\left(\frac{r}{1+r}\right) E_{T} \sum_{i=0}^{\infty}\left(\frac{1}{1+r}\right)^{i} Y_{t+i}
$$

De acordo com a expressão acima, o consumo é uma função da renda permanente, isto é, depende dos ativos financeiros e da expectativa a respeito da renda futura.

\section{REFERÊNCIAS}

BLANCHARD, O.; FISHER, S. Lectures on Macroeconomics. Massachusetts: Institute of Technology, 1989. p. 275-291.

BROWING, R.; LUSARDI, A. Household Saving: Micro Theories And Micro Facts. Journal of Economic Literature, v. xxxiv, p. 1797-1855. Dec. 1996.

DEATON, A. Understanding Consumption. Oxford: Oxford University Press, 1992.

FRIEDMAN, M. A Theory of the Consumption Function. Princeton: Princeton University Press, 1957.

HALL, R. E. Stochastic Implications of the Life-Cycle Permanent Income Hipothesis: Theory and Evidence. Journal of Political Economy, v. 86, n. 6, Dec. 1978. 
KEYNES, J. M. The General Theory of Employment, Interest and Money. London: Macmillan, 1937. (Edição original: 1936).

MODIGLIANI, F. Life Cycle, Individual Thrift, and the Wealth of Nations. American Economic Review, v. 3, Jun. 1986.

NERI, M. O consumidor Keynesiano. Análise Econômica, ano 10, n. 17, p. 37-47, 1992.

ROMER, D. Advanced Macroeconomics. University of California, Berkeley: McGrawHill, 1996.

SACHS, J.; LARRAIN, F. Macroeconomia. São Paulo: Macron Books, 1995. 